\title{
Histomorphometric evaluation of cortical bone surrounding mini-screw: Why is the insertion torque critical for primary stability?
}

\author{
Hiroko Sunagawa-Kojima*, Junji Ohtani, Masato Kaku, Noriko Tsubamoto, Emi Ishikawa, Kazuo \\ Tanne, Kotaro Tanimoto \\ Department of Orthodontics and Craniofacial Developmental Biology, Hiroshima University Graduate School of \\ Biomedical Science, Hiroshima, Japan
}

\begin{abstract}
Objective: Mini-screws have widely been used as a temporary anchorage device. We previously reported the importance of insertion torque for primary implant stability. However, it remains unclear whether or not the destruction of cortical bone surrounding the mini-screw depends on the magnitude of the insertion torque, and whether or not the destroyed bone can be repaired around titanium alloy miniscrews.

Materials and methods: Self-drilling type titanium alloy mini-screws were placed into the rat maxillary bone. Insertion torque and screw diameter were varied and their effect on primary stability of miniscrew was evaluated. Bone remodeling around the mini-screw and the ratio of osteoid and calcified bones was examined using Villanueva bone staining on each section. Moreover, during the experimental period, the tensile strength of the implanted screw was measured.

Results: Irrespective of the diameter of micro-implants which were implanted with 5-10 N.cm insertion torque were in close contact with the cortical bone, whereas the cortical bone was broken when implants were inserted with 15-20 N.cm insertion torque. While using the 15-20 N.cm insertion torque, the screw surface did not exhibit close contact with the cortical bone and osteoid was increased at $56 \mathrm{~d}$ after implantation. Furthermore, tensile strength of the bone in the $15-20 \mathrm{~N}$.cm insertion torque group remained lower than in the 5-10 N.cm group through an experiment period.

Conclusions: Our findings emphasized that we should use an optimal insertion torque at 5-10 N.cm for mini-screw implantation to establish primary stability.
\end{abstract}

Keywords: Mini-screw, Temporary anchorage device (TAD), Bone remodeling, Primary stability.

Accepted on July 26, 2018

\section{Introduction}

Anchorage is one of the most important factors to appropriately control orthodontic tooth movement. Various methods have been introduced for orthodontic anchorage. However, many of them depend on patient compliance [1-3]. In clinical orthodontics, temporary anchorage devices that anchor tooth movement on the alveolar and jaw bones, have recently received much attention [4-6]. Consequently, various mini-screws have now been developed for reinforced anchorage in orthodontic tooth movement with much less patient compliance.

We have already reported that cortical bone is broken by excessive insertion torque, resulting in significant loss of primary stability [7]. However, it is still unclear whether or not the damaged bone can be repaired around mini-screws.

Certain consensus has been obtained by previous studies of dental implant [8-10], that the healing time after implantation is important for stable bone remodeling leading to high success rate. Experimental studies have also demonstrated that pure titanium mini-screws used for orthodontic anchorage can be immediately loaded by occlusal force without any reduction in stability [11-13]. Roberts et al. [14] suggested that a $6 \mathrm{w}$ healing period is adequate for implants to resist orthodontic forces in rabbits. In addition, Deguchi et al. [15] showed that bone remodeling and osseointegration were not impaired by orthodontic loading once the screw was implanted into the bone. However, in the case of self-drilling type titanium alloy mini-screws, it still remains unclear whether or not bone remodeling enough to enhance stability of mini-screws occurs around the implant surface.

The aim of this study was to evaluate the condition of the cortical bone surrounding mini-screws and to examine optimal insertion torque in the aspect of the bone remodeling. Therefore, in this study, we examined the influence of screw diameter and insertion torque on the stability of titanium alloy mini-screws, and the tensile strength of bone around the miniscrews during healing period. 


\section{Materials and Methods}

\section{Surgical procedures}

Self-drilling type mini-screws (Dual-top anchor screw, Jeil Medical Co., Seoul, Korea), $1.6 \mathrm{~mm}$ and $2.0 \mathrm{~mm}$ in diameter and $6.0 \mathrm{~mm}$ in length, made of titanium alloy (Ti6Al4V alloy) were used. Fifteen-week-old Wistar male rats (Charles River Laboratories Japan, Inc., Yokohama, Japan) were used in this study. Following implantation, the healing period was defined as 7, 28, or $56 \mathrm{~d}$. Eighty animals were used and five animals were assigned to each experimental group.

Mini-screws were placed $2 \mathrm{~mm}$ distal to the upper incisors, and the insertion angle was adjusted to $90^{\circ}$ to the cortical bone surface (Figure 1A). All mini-screws were inserted using the self-drilling method and divided into the following two groups by adjusting the pressure force: high driving torque group (HDT group: 15-20 N.cm) and low driving torque group (LDT group: 5-10 N.cm). At the end of implantation, we measured the insertion torque using an industrial hand driver (FTD20CNS, TOHNICHI, Ltd., Tokyo, Japan). Immediately and 7, 28, and $56 \mathrm{~d}$ after implantation, the rats were scarified, and the maxillary bone surrounding the micro-implant was resected and examined.

\section{Histomorphometric examination}

Rat maxillary bone specimens were fixed with $70 \%$ ethanol for more than one day for Villanueva bone staining (Wako Pure Chemical Industries, Ltd., Osaka, Japan), and subsequently embedded in methylmetacrylate (Technovit ${ }^{\circledR} 7200$, Kulzer Co., Hanau, Germany). The embedded specimens were cut along the long axis of mini-screw by a micro cutting machine (BS3000, EXAKT Co., Remscheid, Germany) and grinded into $20 \mu \mathrm{m}$ sections by a grinding machine (SBT900, South Bay Technologies, San Clemente, CA, USA) (Figures 1B and 1C). Bone remodeling around the mini-screw was observed using a fluorescence microscope (BZ-8100, KEYENCE Co., Osaka, Japan).

An analysis area of $1000 \times 2000 \mu \mathrm{m}$ was defined on each section as illustrated by areas A and B in Figure 1D. Area A was on the micro-implant surface and area B was $1000 \mu \mathrm{m}$ away from area A (Figures $1 \mathrm{E}$ and $1 \mathrm{~F}$ ). Villanueva staining solution detected calcified and osteoid bones surrounding the mini-screw. Under the fluorescence microscope, calcified and osteoid bones can be distinguished by green or jade green and red colors, respectively. Fluorescence intensity was quantified using analysis software (BZ-II analysis application, KEYENCE Co.), and the luminance values of calcified and osteoid bone were used to calculate the osteoid bone/ calcification bone ratio $(\mathrm{O} / \mathrm{C}$ ratio). We used the $\mathrm{O} / \mathrm{C}$ ratio to evaluate bone remodeling in the cortical bone surrounding mini-screws. High $\mathrm{O} / \mathrm{C}$ ratio values indicated increased osteoid bone formation and active remodeling.
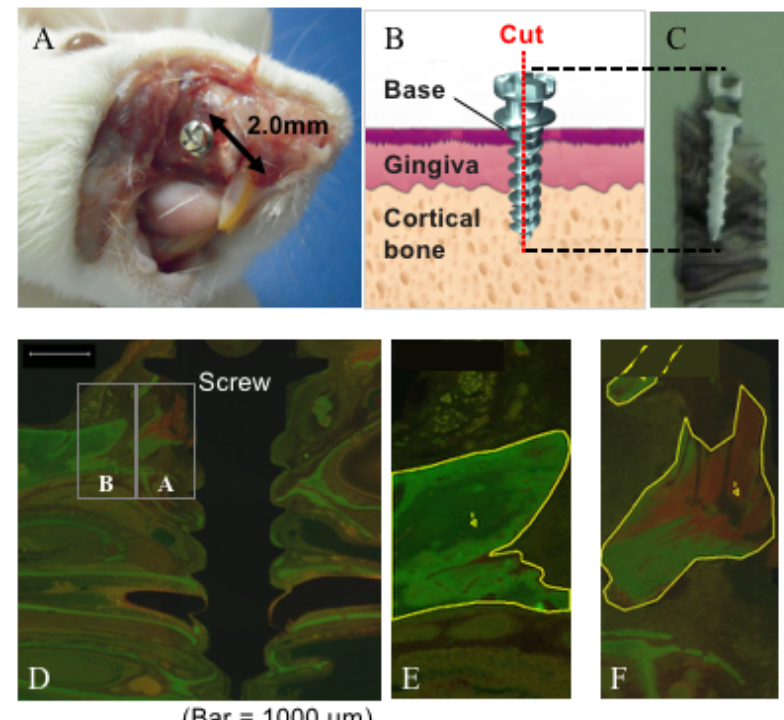

Area B

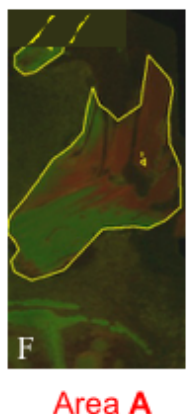

Figure 1. Mini-screw placed $2.0 \mathrm{~mm}$ distal to the upper incisors (A). Mini-screw was cut along the long axis (B). Schema of implantation of mini-screw ( $C$ and D). The Villanueva staining embedded in metylmetacrylate $(D)$. Area $B$ : area of $1000 \times 2000 \mu \mathrm{m}$, apart from area $A(E)$. Area A: area of $1000 \times 2000 \mu \mathrm{m}$, close to the implant body surface $(F)$.

\section{Tensile strength test}

Rat maxillary bone specimens were fixed with $70 \%$ ethanol for more than one day and were firmly fixed with high strength dental stone (NEW FUJIROCK, GC, Tokyo, Japan). Then, the mini-screw was pulled to the vertical direction against the implanting direction to measure the tensile strength by tensile test machine (EZ-TEST, Shimadzu Co., Kyoto, Japan) (Figure 2).

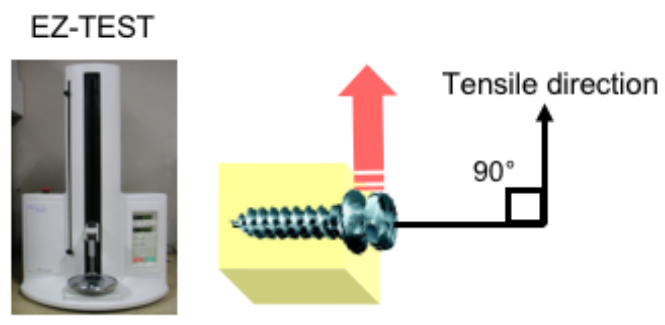

Figure 2. Mini-screw was pulled to the vertical direction against the implanting direction to measure the tensile strength by use of a tensile test machine.

\section{Statistical analysis}

Statistical analysis was performed using analysis of variance and a multiple-comparison test (Fisher's). Confidence levels of $\mathrm{p}<0.05$ and $\mathrm{p}<0.01$ were defined as statistically significant. 
Histomorphometric evaluation of cortical bone surrounding mini-screw: Why is the insertion torque critical for primary stability?

\section{Results}

\section{Histomorphometric findings}

In LDT group of both 1.6 and $2.0 \mathrm{~mm}$ diameter, the cortical bone was not broken immediately after the implantation. The mini-screws and cortical bone were in close contact at the interface (Figures $3 \mathrm{~A}$ and $3 \mathrm{~B}$ ). Close contact of the cortical bone to the mini-screw surface was maintained $56 \mathrm{~d}$ after the implantation (Figures 3C and 3D).
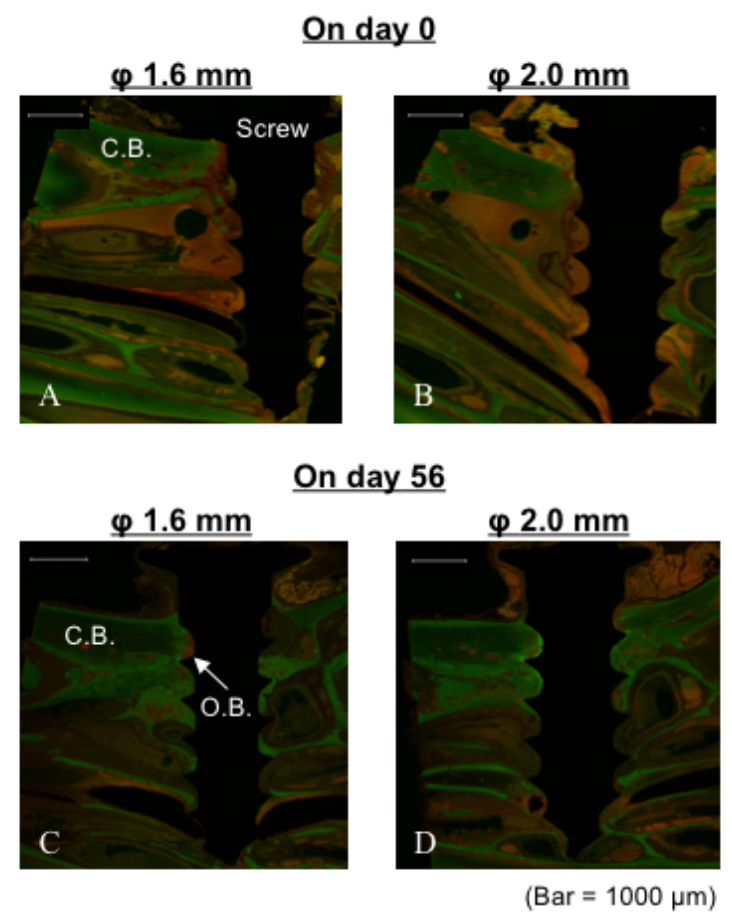

Figure 3. On the photomicrograph, mineralized, immature mineralized and osteoid bone (O.B.) appeared in green, jade green, and red-brown colors, respectively.

In HDT group of both 1.6 and $2.0 \mathrm{~mm}$ diameter, the cortical bone was not broken immediately after the implantation. The mini-screws and cortical bone were in close contact at the interface (Figures 4A and 4B). However, cortical bone around mini-screws was broken $56 \mathrm{~d}$ after implantation (Figures $4 \mathrm{C}$ and 4D).

Immature mineralized bone was observed as a jade green color and some unmineralized osteoid bone was observed in the cortical bone area directly in contact with the mini-screw surface in LDT group (Figure 5). These findings were observed regardless of the mini-screw diameter. On the other hand, the mini-screw surface did not come into close contact with the cortical bone in HDT group. These phenomena were observed after implantation of both 1.6 and $2.0 \mathrm{~mm}$ diameter miniscrews.

\section{O/C ratio}

In both 1.6 and $2.0 \mathrm{~mm}$ diameter LDT groups, the $\mathrm{O} / \mathrm{C}$ ratio peaked at around 1.0 on $\mathrm{d} 7$ in area $\mathrm{A}$ (Figures $6 \mathrm{~A}$ and $6 \mathrm{~B}$ ).
The $\mathrm{O} / \mathrm{C}$ ratio in $1.6 \mathrm{~mm}$ diameter LDT group was significantly $(p<0.01)$ higher on $d 7$ than that on $d 0$. The $O / C$ ratio in HDT group with $1.6 \mathrm{~mm}$ diameter mini-screws was significantly higher than that in LDT group on $\mathrm{d} 0$ and 56 $(p<0.05$ and $p<0.01$ respectively).
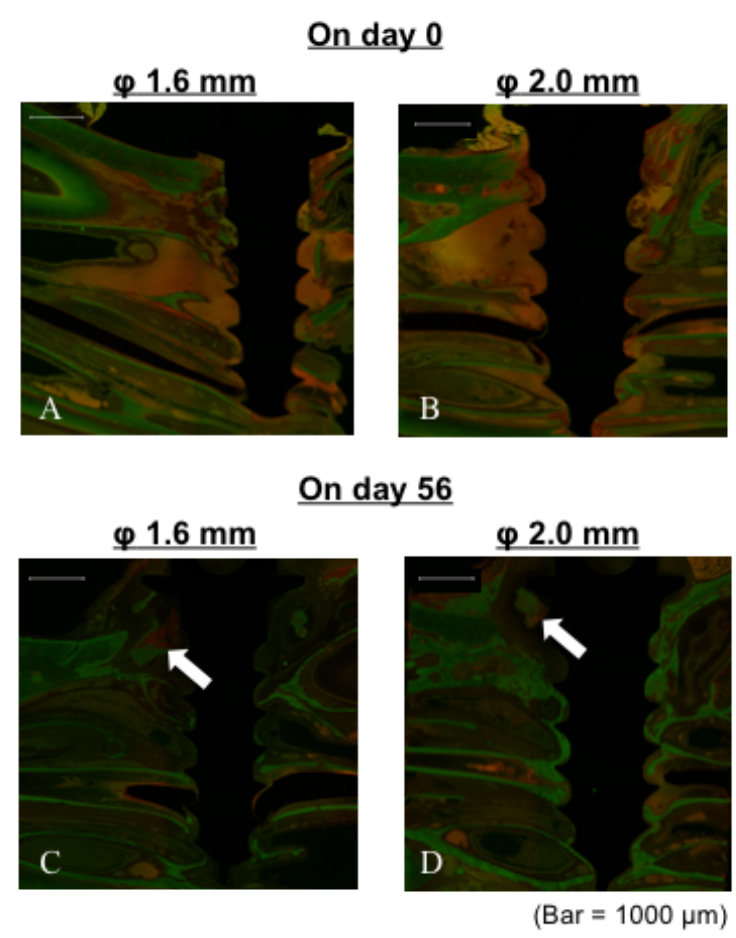

Figure 4. On the photomicrograph, mineralized, immature mineralized and osteoid bone appeared green, jade green, and redbrown colors, respectively.

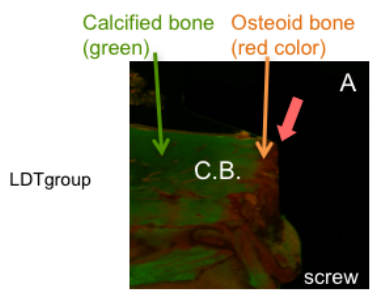

On day 0

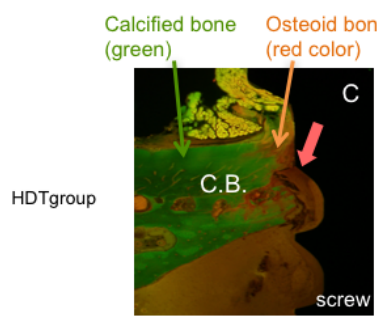

On day 0

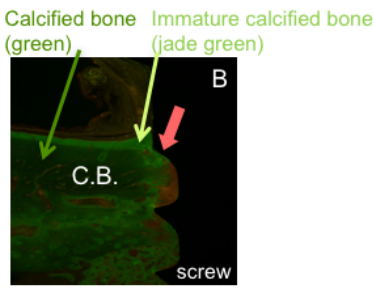

On day 56

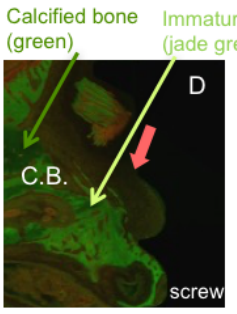

On day 56

Figure 5. In LDT group, osteoid bone at the surface of the mini-screw on $d 0$ replaced immature calcified bone on d 56 (A and B). In HDT group, the broken cortical bone did not come into close contact with the mini-screw surface on 56 (C and D).

On the other hand, the $\mathrm{O} / \mathrm{C}$ ratio in LDT group with $1.6 \mathrm{~mm}$ diameter mini-screws was significantly $(p<0.05)$ higher than 
that in HDT group on $\mathrm{d} 7$ (Figure 6A). A significantly $(\mathrm{p}<0.05)$ higher $\mathrm{O} / \mathrm{C}$ ratio was observed $28 \mathrm{~d}$ after implantation of 2.0 $\mathrm{mm}$ diameter mini-screws in HDT group as compared with LDT group (Figure 6B). In HDT group, the highest $\mathrm{O} / \mathrm{C}$ ratio was observed on $\mathrm{d} 28$, irrespective of the diameter.

The $\mathrm{O} / \mathrm{C}$ ratio was significantly $(\mathrm{p}<0.05)$ higher on $\mathrm{d} 28$ than $\mathrm{d}$ 0 in area $\mathrm{B}$, regardless of the screw diameter and driving torque (Figures 6C and 6D). Bone remodeling was almost completed on $\mathrm{d} 56$ because the $\mathrm{O} / \mathrm{C}$ ratio was similar in all groups immediately after implantation. Both in LDT group and HDT group, differences in diameter had no substantial influence on the $\mathrm{O} / \mathrm{C}$ ratio.
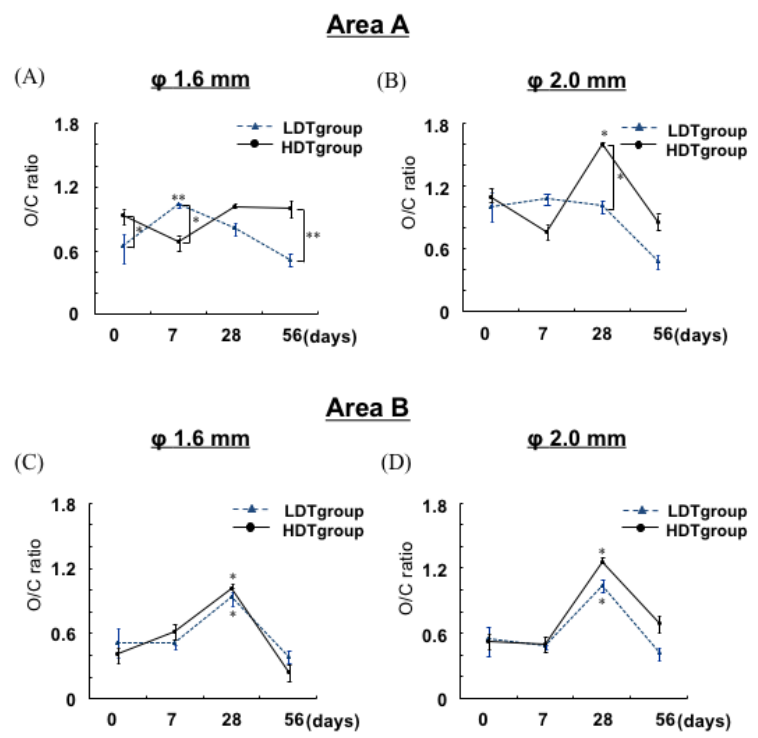

Figure 6. (A) The O/C ratio of LDT group was significantly higher on $d 7$ than on $d 0$ in area $A$. The $O / C$ ratio was significantly higher in $H D T$ group than in LDT group on $d 0$ and 56. The $O / C$ ratio was significantly higher in LDT group than in HDT group on $d$ 7. (B) The $O / C$ ratio of HDT group was significantly higher on $d 28$ than $d 0$ in area $A$. The $O / C$ ratio was significantly higher in HDT group than in LDT group on $d 28$. (C and D) Regardless of the screw diameter and the magnitude of insertion torque, the $\mathrm{O} / \mathrm{C}$ ratio was significantly higher on $d 28$ than on $d 0$ in area $B$. (For the time course assessment: ${ }^{*} p<0.05$ and ${ }^{* *} p<0.01$ vs. 0 day).
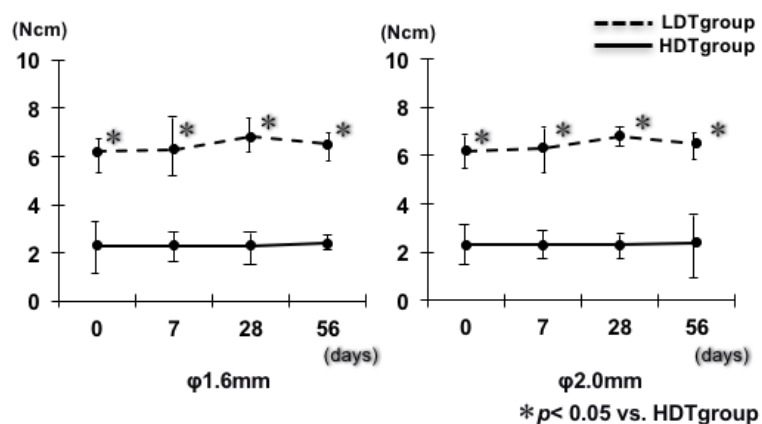

Figure 7. Tensile strength of mini-screw was significantly greater in LDT group than HDT group during healing period.

\section{Tensile strength test}

Irrespective of the mini-screw diameter, tensile strength of bone around mini-screw in LDT group was significantly $(p<0.05)$ greater than that in HDT group through the experimental period (Figure 7). Tensile strength of mini-screw in each group showed no significant differences up to $56 \mathrm{~d}$ after the implantation irrespective of the insertion torque. Thus, during healing period, tensile strength of the bone after implantation of titanium alloy self-drilling screw showed no changes in all the experimental groups.

\section{Discussion}

Peri-implant inflammation usually occurs after implantation and is followed by bone remodeling [16]. Bone healing occurs in the following three stages: an inflammatory stage, a fibroblastic stage, and a remodeling stage [17]. Bone remodeling is a continuous process of bone resorption and formation [18,19]. After implantation, inflammatory cells infiltrate the surrounding tissue and osteoclasts initiate bone resorption. This is followed by bone formation by osteoblasts to achieve osseointegration [20]. Bone healing should occur before soft tissue healing for successful osseointegration. If soft tissue healing starts before bone remodeling, fibrous soft tissue will invade the interface between the implant and surrounding bone. Healing in soft tissues (9-day turn-over) is faster as compared with bony tissues (2-4 month turn-over) [21].

In this study, the mini-screw surface was in close contact with the cortical bone after implantation with an insertion torque of 5-10 N.cm. This finding demonstrated that close contact was maintained between the bone and mini-screw after insertion of the mini-screw under the appropriate insertion torque. Miniscrew inserted with an insertion torque of 15-20 N.cm did not come into contact with the cortical bone even $56 \mathrm{~d}$ after implantation, and the $\mathrm{O} / \mathrm{C}$ ratio in the HDT group reached a peak after implantation later than LDT group. This may be caused by soft tissue invasion of the gap between the cortical bone and mini-screw because soft tissue healing is faster than bony tissue healing. In addition, regardless of the areas $\mathrm{A}$ and $\mathrm{B}, \mathrm{O} / \mathrm{C}$ ratios were the highest $28 \mathrm{~d}$ after implantation in HDT group, indicating that the fragile osteoid bone surrounding the mini-screw increased in a wide range. Thus, it was speculated that the stability of mini-screw in HDT group indicated low values compared with that in LDT group. Since alveolar bone thickness is similar in rats and humans $(1.0 \mathrm{~mm}$ in rats, $1-1.5$ $\mathrm{mm}$ in humans) [22,23], the appropriate insertion torque (in the range of 5-10 N.cm) would be critical for the stable insertion of mini-screws also in human alveolar bone.

The top and rear half of the mini-screws used in this study had different structures. The top half had a cylindrical structure and the rear half a tapered structure. Therefore, after the rear half was self-drilled into the cortical bone, the insertion torque was assumed to increase prominently. Clinicians should check the implications of mini-screw structure at insertion. It is recommended to control insertion torque not to exceed the 
optimal range. Bone-Implant-Contact (BIC) is important for mini-screw stability. Ohtani et al. [7] reported that BIC was significantly higher when implants were inserted with 5-10 N.cm insertion torque than with $15-20$ N.cm insertion torque, irrespective of the driving method. Consequently, it was necessary to consider magnitude of insertion torque. When the high insertion torque beyond the range is applied to the bone around mini-screw primary stability of mini-screw would be reduced.

It was reported that the $\mathrm{O} / \mathrm{C}$ ratio reflects bone remodeling around the mini-screw after implantation and the osteoid bone was remodeled around the mini-screw by osteoclasts and osteoblasts [24]. Increased osteoid bone formation accelerates bone remodeling, and the process of bone remodeling is completed by the formation of lamellar bone [24]. Based on our previous report [7], it became clear that the condition of the contact between cortical bone and mini-screw affects initial stability, and trabecular bones surrounding screw are not tough enough to contribute to the mini-screw stability. Therefore, in this study, the $\mathrm{O} / \mathrm{C}$ ratio was measured the cortical bone.

In this study, we examined bone remodeling around the miniscrew and we proved that when the mini-screw was implanted with excessive insertion torque, cortical bone was destroyed and followed by active remodeling. If the mini-screw is implanted with optimal insertion torque, then bone remodeling may progress with close contact to mini-screw. Tensile strength showed high value in LDT group through an experiment period, suggesting that immediate loading of orthodontic force would be possible when appropriate insertion torque is applied. In conclusion, insertion torque is suggested to be a critical factor for the primary stability of mini-screws. These findings were observed regardless of the mini-screw diameter.

\section{Acknowledgments}

I would like to thank to Dr. Kazuhiro Ueki for his kindest guidance and support. This investigation was supported in part by Grant-in Aid for Scientific Research of Japan Society for the Promotion of Science (19890139).

\section{References}

1. Gabriel HF. Motivation of the headgear patient. Angle Orthod 1968; 38: 129-135.

2. Egolf RJ, BeGole EA, Upshaw HS. Factors associated with orthodontic patient compliance with intraoral elastic and head gear wear. Am J Orthod Dentofacia Ortho 1990; 97: 336-348.

3. Melsen B, Dalstra M. Distal molar movement with Kloehn headgear: Is it stable? Am J Orthod Dentofacial Orthop 2003; 123: 374-378.

4. Kanomi R. Mini-implant for orthodontic anchorage. J Clin Orthod 1997; 31: 763-767.

5. Lee JS, Park HS, Kyung HM. Micro-implant anchorage for lingual treatment of a skeletal Class II malocclusion. J Clin Orthod 2001; 35: 643-647.
6. Park HS, Bae SM, Kyung HM, Sung JH. Micro-implant anchorage for treatment of skeletal Class I bialveolar protrusion. J Clin Orthod 2001; 35: 417-422.

7. Ohtani J, Sunagawa H, Fujita T, Kawata T, Kaku M, Tohma Y. Stability of mini-screw as an implant anchorage placed onto rat maxilla. Orthod Waves Jpn Ed 2008; 67: 125-131.

8. Futami T, Fujii N, Ohnishi H, Taguchi N, Kusakari H, Ohshima H. Tissue response to titanium implants in the rat maxilla: ultrastructural and histochemical observations of the bone-titanium interface. J Periodontol 2000; 71: 287-298.

9. Covani U, Bortolaia C, Barone A, Sbordone L. Buccolingual crestal bone changes after immediate and delayed implant placement. J Periodontol 2004; 75: 1605-1612.

10. Palmer RM, Palmer PJ, Baker P. Immediate and early replacement implants and restorations. Dent Update 2006; 33: 262-264, 266-268.

11. Majzoub Z, Finottti M, Miotti F, Giardino R, Aldini NN, Cordioli G. Bone response to orthodontic loading of endosseous implants in the rabbit calvaria: early continuous distalizing forces. Eur J Orthod 1999; 21: 223-230.

12. Buchter A, Wiechmann D, Koerdt S, Wiesmann HP, Piffko J, Meyer U. Load-related implant reaction of miniimplants used for orthodontic anchorage. Clin Oral Impl Res 2005; 16: 473-479.

13. Berens A, Wiechmann D, Dempf R. Mini- and microscrews for temporary skeletal anchorage in orthodontic therapy. J Orofac Orthop 2006; 67: 450-458.

14. Roberts WE, Smith RK, Zilberman Y, Mozsary PG, Smith RS. Osseous adaptation to continuous loading of rigid endosseous implants. Am J Orthod 1984; 86: 95-111.

15. Deguchi T, Takano-Yamamoto T, Kanomi R, Hartsfield JK, Roberts WE, Garetto LP. The use of small titanium screws for orthodontic anchorage. J Dent Res 2003; 82: 377-381.

16. Ito M, Shimoda S, Sato J. A morphological investigation of bone healing (rabbit tibia) at the interface with dental implant. Tsurumi Univ Dent J 2005; 31: 81-99.

17. Fujii N, Kusakari H, Maeda T. A histological study on tissue responses to titanium implantation in rat maxilla: the process of epithelial regeneration and bone reaction. $\mathrm{J}$ Periodontol 1998; 69: 485-495.

18. Misch CE. Implant design considerations for the posterior regions of the mouth. Implant Dent 1999; 8: 376-386.

19. Misch CE, Bidez MW, Sharawy M. A bioengineered implant for a predetermined bone cellular response to loading forces. A literature review and case report. J Periodontol 2001; 72: 1276-1286.

20. Haga M, Fujii N, Nozawa-Inoue K, Nomura S, Oda K, Uoshima K. Detailed process of bone remodeling after achievement of osseointegration in a rat implantation model. Anat Rec (Hoboken) 2009; 292: 38-47. 
21. Vignery A, Baron R. Dynamic histomorphometry of alveolar bone remodeling in the adult rat. Anat Rec 1980; 196: 191-200.

22. Deguchi T, Nasu M, Murakami K, Yabuuchi T, Kamioka H, Takano-Yamamoto T. Quantitative evaluation of cortical bone thickness with computed tomographic scanning for orthodontic implants. Am J Orthod Dentofacial Orthop 2006; 129: 721-732.

23. Gracco A, Luca L, Cozzani M, Siciliani G. Assessment of palatal bone thickness in adults with cone beam computerized tomography. Aust Orthod J 2007; 23: 109-113.

24. Zhang L, Zhao $\mathrm{Z}$, Li Y, Wu J, Zheng L, Tang $\mathrm{T}$. Osseointegration of orthodontic micro-screw after immediate and early loading. Angle Orthod 2010; 80: 354-360.

\section{*Correspondence to}

Hiroko Sunagawa-Kojima

Department of Orthodontics and Craniofacial Developmental Biology

Hiroshima University Graduate school of Biomedical Science

Hiroshima

Japan 